ELASTICITY OF COPPER AND STEEL AT - I86 C.

By J. R. Benton.

KNOWLEDGE of the dependence of the elasticity of solids on temperature, aside from any scientific value in itself, is of interest also because inferences can be drawn from it regarding the variation of other mechanical and thermal properties, ${ }^{1}$ and because of its possible bearing on the theory of intermolecular forces in solids.

The variation of elasticity with temperature has been studied by Wertheim, ${ }^{2}$ Kohlrausch and Loomis, ${ }^{3}$ Kupffer, ${ }^{4}$ Streintz ${ }^{5}$ and Kiewiet, ${ }^{6}$ and was the subject of very careful researches by Pisati. ${ }^{7}$ These investigators worked mostly above ordinary temperature; Wertheim extended his experiments to $-{ }_{1} 5^{\circ} \mathrm{C}$; t the others did not go below $0^{\circ}$. The extremely thorough experiments of Pisati, which furnish such complete and accurate information ${ }^{8}$ for the substances and temperatures he used, were made between $20^{\circ}$ and $300^{\circ} \mathrm{C}$.

${ }^{1}$ See article: Thermodynamic Formulæe for Isotropic Solids subject to Tension in one Direction, Phys. Rev.

${ }^{2} \mathrm{G}$. Wertheim : Untersuchungen über die Elastizität. Pogg. Ann., Ergänzungsband II., pp. I-I I 5, I 848 .

${ }^{3}$ F. Kohlrausch and Loomis: Pogg. Ann.. CXLI., p. 48r, 1870

${ }^{4}$ A. Kupffer: Mém. de l'Acad. imp. de St. Petersbourg (VI. sér.), Vol. VI , p. 393, 1857.

${ }^{5}$ H. Streintz : Über die Änderung der Elastizität und der Länge eines vom galvanischen Strome durchflossenen Drahtes. Pogg. Ann., CL., p. 368, I873.

${ }^{6} \mathrm{~J}$. Kiewiet : Wiedermann's Ann., XXIX., 6r 7-654, 1886.

${ }^{7}$ G. Pisati : Sulla Elasticita dei Metalli a diverse Temperature. Nuovo Cimento, 3d ser., Vol. I., p. I8I ; Vol. II., p. I37, 1877; Vol. IV., p. 152, 1878; Vol. V., pp. $34, \mathrm{I} 35, \mathrm{I} 45, \mathrm{I} 879$.

8 One criticism may be made on Pisati's experiments. He employed the method of torsional oscillations, using wires about $70 \mathrm{~cm}$. long; for such short wires the uncertain distribution of stress at the points of support may have an influence appreciable within his limits of error. 
The low temperatures now available for scientific work make possible an extension of this study of elasticity as influenced by temperature, previously carried on above, but not below, ordinary temperature.

Some of the mechanical properties of matter have already been investigated at liquid air temperature. The ultimate tensile strength of a number of metals was measured by Dewar, ${ }^{1}$ who found it greatly increased in most cases, the increase of strength being accompanied by decreased elongation at rupture; decreased elongation appears to accompany the increased strength due to low temperature, much as it accompanies at ordinary temperature the increased strength in iron and steel, due to the presence of other substances in chemical combination or as alloys.

Dewar made no quantitative determinations of the elastic moduli, however, although he mentions several experiments which indicate an increase of elasticity at low temperature. Thus a rod of fusible metal, supported at one end and loaded at the other, offered such resistance to flexure as to indicate a value of Young's modulus four or five times that at ordinary temperature. A spiral of fusible metal wire, which would be pulled straight by an ounce weight at ordinary temperature, supported two pounds at liquid air temperature, and vibrated like a steel spring ; thus showing greatly increased modulus of rigidity. Dewar attempted to measure the rigidity modulus of steel by means of a spiral spring, but could detect no change; my experiments show a slight increase. The greatly increased rigidity of rubber is familiar to every one who has used liquid air.

There is no reason, however, to expect that most substances increase in elasticity at liquid air temperature as much as fusible metal or rubber. The metals of Pisati's experiments without exception decreased in rigidity with rising temperature, but at a rate that increassd with the temperature. The change of rigidity with temperature is then less as a solid is further from its melting point. Furthermore, Pisati's results for different substances at the same temperature show in general a smaller rate of change, as the sub-

1 James Dewar: Scientific Uses of Liquid Air. Proc. Roy. Inst., Vol. XIV., pp. 393-404, 1894 . 
stance is farther from its melting point. ${ }^{1}$ If we may extrapolate from Pisati's results, we should expect a substance like iron which melts about $\mathrm{I} 600^{\circ} \mathrm{C}$., to increase much less in rigidity at liquid air temperature than Dewar's fusible metal, whose melting point may have been below $200^{\circ} \mathrm{C}$.

This reasoning appears confirmed by direct measurement of the elasticity of copper and steel wires at liquid air temperature.

\section{Results of ExPERIMENTS.}

Each of the numerical values about to be submitted represents only a single complete determination. ${ }^{2}$ Unavoidable circumstances prevented continuing the experiments so as to verify by repeated trials the results obtained, or to make determinations with other substances than copper and steel. It seems worth while, however, to put the experiments on record, even though the results are not numerous, and can not claim the same reliability as if there had been an opportunity to repeat the determinations.

The numerical values given below were obtained from wires approximately one meter long and $1.5 \mathrm{~mm}$. in diameter. Young's modulus was determined by micrometer measurement of the extension under known tension; the modulus of rigidity by measurement of the statical twist due to a known torque; a detailed description of the apparatus follows later. In determining Young's modulus, the tension was varied approximately from 3 to I I kgs. weight per square $\mathrm{mm}$. For the rigidity modulus, the torque was varied from

${ }^{1}$ I have computed the accompunying table from Pisati's values: the first column of figures gives the melting point, the second gives the change in rigidity modulus per degree Celsius at $0^{\circ}$, expressed in percentage of rigidity modulus at $0^{\circ} \mathrm{C}$; that is, $\frac{100}{R_{0}} \cdot \frac{\partial R}{\partial T}$, where $R$ is the modulus of rigidity, and $T$ the temperature.

$\begin{array}{lll}R_{0} & 600^{\circ}-650^{\circ} \mathrm{C} . & 0.064 \\ \text { Aluminum, } & 910 & 0.035-0.039 \\ \text { Brass, } & 920-1040 & 0.027-0.041 \\ \text { Silver, } & 1035-1250 & 0.0204 \\ \text { Gold, } & 1050-1330 & 0.023-0.025 \\ \text { Copper, } & 1300-1400 & 0.0195 \\ \text { Steel, } & 1500-1800 & 0.0207 \\ \text { Iron, } & 1765-2200 & 0.012-0.015\end{array}$

2 Except that of Young's modulus for copper, which was determined twice with the same wire. The two results differed by 3 per cent. 
IOO to 300 dyne-centimeters per centimeter of length, the (constant) tension being about I I kgs. weight per square mm.

Denoting by $Y_{T}$ and $R_{T}$ Young's modulus and the rigidity modulus at the temperature $T^{\circ} \mathrm{C}$, , the experiments give the following results :

For the copper wire (hard-drawn), making no allowance for thermal contraction,

$$
\begin{aligned}
& Y_{-186}=Y_{24} \times 1.177 \pm 0.020,{ }^{1} \\
& R_{-186}=R_{21} \times 1.063 \pm 0.003
\end{aligned}
$$

For the steel wire (piano wire),

$$
\begin{aligned}
& Y_{-186}=Y_{21} \times 1.085 \pm 0.007, \\
& R_{-186}=R_{20} \times 1.072 \pm 0.006 .^{2}
\end{aligned}
$$

By correcting for thermal contraction, these values are slightly modified; but the change in them is of the same order as the probable errors, so that it is not necessary to know the coefficients of thermal expansion at low temperatures with extreme accuracy, provided we can be sure they do not increase greatly with decreasing temperature. There is no reason to expect such an increase. The experiment of Zakrzewski ${ }^{3}$ extending to $-103^{\circ} \mathrm{C}$., show diminished coefficient of expansion with decreasing temperature. By extrapolation from his results the average coefficient of expansion of copper between $20^{\circ}$ and $-186^{\circ}$ may be taken as 0.0000156 , and that of iron as 0.0000105. Using these values, we obtain the corrected ratios :

For the copper wire :

$$
\begin{aligned}
& Y_{-186}=Y_{24} \times 1.180, \\
& R_{-186}=R_{21} \times 1.073 .
\end{aligned}
$$

For the steel wire

$$
\begin{aligned}
& Y_{-186}=Y_{21} \times 1.087 . \\
& R_{-186}=R_{20} \times 1.079 .
\end{aligned}
$$

${ }^{1}$ The \pm refers only to the accidental errors, and takes no account of the systematic error mentioned on page 24 .

2 These ratios refer to the values at room temperature before cooling; those after cooling differed slightly. See page 23 .

${ }^{3} \mathrm{~J}$. Zakrzewski : Der Ausdehnungskoeffient fester Körper bei tiefen Temperaturen. Bull. de l'Acad. de Cracow, 1889. Abstract in Die Fortschritte der Physik, I889. 
According to the theory of elasticity, Poisson's ratio $(x)$ is expressible in terms of Young's modulus and the modulus of rigidity by the formula ${ }^{1}$

$$
x=\frac{Y-2 R}{2 R} .
$$

From this equation we find for the copper wire $x_{-186}=1.098 x_{20}$ +0.098 ; and for the steel wire $x_{-186}=1.008 x_{20}+0.008$. Poisson's ratio then is greater than at ordinary temperature.

\section{Comparison with the Value of Elasticity at Other TEMPERATURES.}

The mechanical properties of matter in the solid state vary with different specimens of the same substance. Accordingly our numerical values, obtained from a single copper wire and a single steel wire, must be considered as representing simply the behavior of those two particular wires, and not that of copper and steel in general; these values can at best furnish but rough indications of what may be expected with other copper and steel wires. This is more particularly the case because the wires did not undergo special treatment to reduce them to normal elastic condition, such as repeated heating and cooling, which would have been too wasteful of liquid air.

Nevertheless it is interesting to compare the variation of elasticity in these wires with that observed in other wires at higher temperatures by Pisati and Wertheim. I have accordingly plotted some of the values obtained by these observers, together with my own for liquid air temperature (Figs. I-4, page 22). Regarding the modulus of rigidity, however, it must be remembered that in my experiments torsion took place isothermally, while in Pisati's it must have taken place nearly adiabatically, as the period of torsional oscillations was less than two seconds.

It will be seen that my results are not greatly different from those which would be obtained by extrapolation from the values of Pisati and Wertheim, except in the case of Young's modulus for steel, which is considerably greater at $-186^{\circ}$ than would be expected

1 This formula assumes homogeneity, an assumption which is but imperfectly fulfilled in drawn wires. 


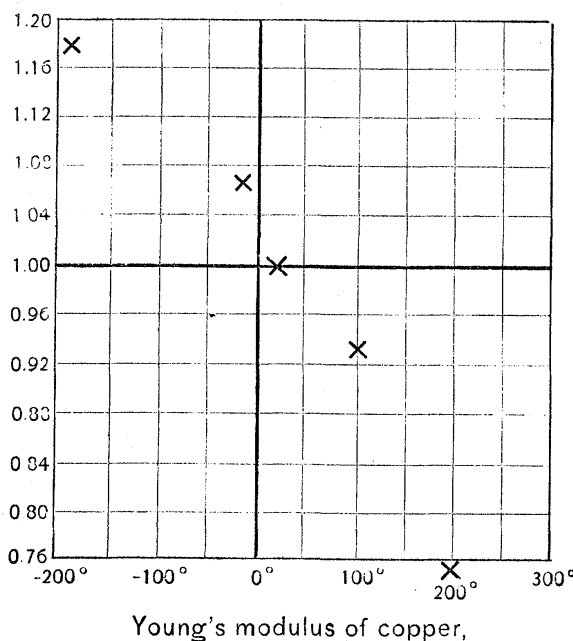

in terms of its value at $20^{\circ} \mathrm{C}$

Wertheim's results for high temperatures.

My result for $-186^{\circ} \mathrm{C}$.

Fig. 1.

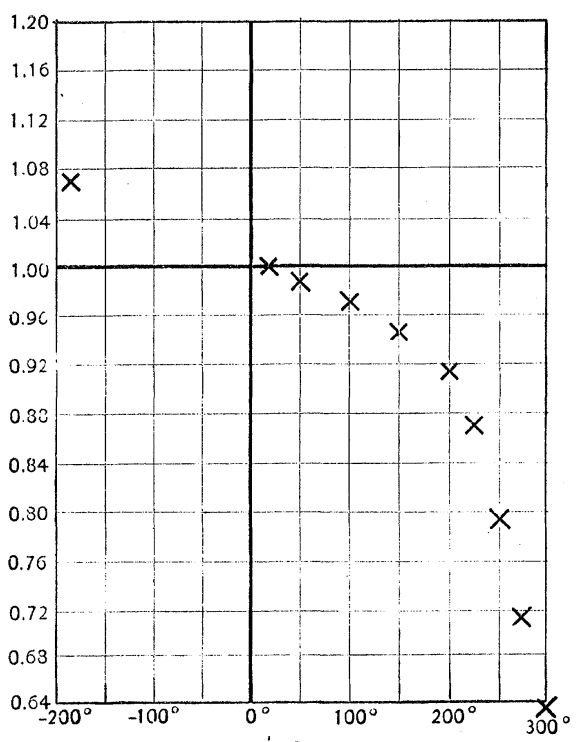

Rigidity modulus of hard-drawn copper in terms of its value at $20^{\circ} \mathrm{C}$.

Pisati's values for high temperatures. My result for $-186^{\circ} \mathrm{C}$.

Fig. 3.

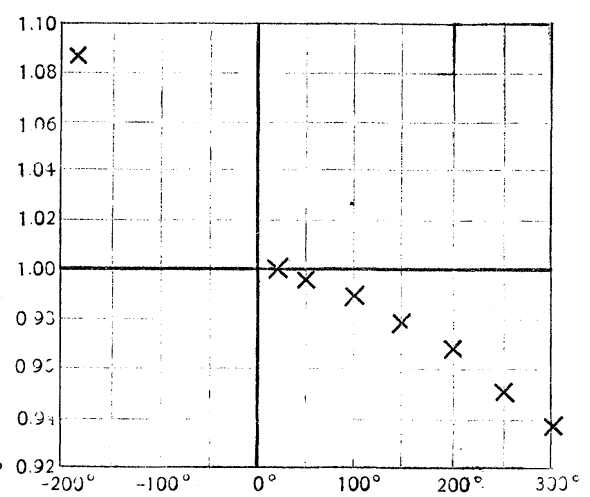

Young's modulus of steel,

in terms of its value at $20^{\circ} \mathrm{C}$.

Pisati's results for high temperatures.

$$
\text { My result for }-186^{\circ} \mathrm{C} \text {. }
$$

Fig. 2.

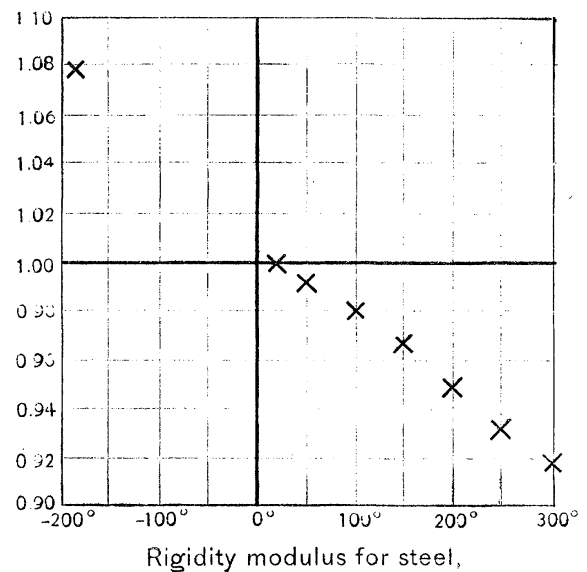

in terms of its value at $20^{\circ} \mathrm{C}$

Pisati's results for high temperatures.

My result for $-186^{\circ} \mathrm{C}$

Fig. 4. 
from Pisati's experiments. My result, corresponding to an average increase of about 0.04 per cent. per degree C., seems more in accordance with the rather vague statement of Styffe, ${ }^{1}$ that Young's modulus for steel increases with decreasing temperature not more than 0.03 per cent., or at most 0.05 per cent. per degree $C$.

\section{Annealing Effect of Liguid Air.}

Wertheim ${ }^{2}$ observed that subjecting metals to low temperature $\left(-15^{\circ} \mathrm{C}\right.$.) leaves a residual effect, similar to that due to annealing when they are heated. The same phenomenon was observable in my experiments. Thus the modulus of rigidity of the copper wire after cooling to $-186^{\circ}$ and warming again to room temperature, was less than it had been before, in the ratio of $0.974 \pm 0.003: \mathrm{I}$, while that of the steel wire was greater in the ratio of $1.022 \pm 0.005: 1$. Sudden cooling from high to ordinary temperature anneals copper and hardens steel; it appears that the same effect occurs in copper as a result of sudden cooling from ordinary to very low temperature, since the modulus of rigidity of copper decreases with annealing. ${ }^{3} \quad$ From the general impression that the modulus of rigidity of steel is increased by hardening, it would be easy to conclude also that steel is hardened by sudden cooling to $-186^{\circ}$; recent experiments, however, show that the rigidity is slightly decreased, instead of increased, by hardening. ${ }^{4}$ From this it would appear that steel is not hardened, but annealed, by sudden cooling from ordinary temperature; but the data are not sufficient to draw general conclusions.

Description of the Apparatus for Young's Modulus.

The arrangement used to determine Young's modulus is shown in Fig. 5, which is not drawn to scale, but is simply a diagram; some of the dimensions being exaggerated, and front and side elevations being combined in one figure.

\footnotetext{
${ }^{1}$ Knut Styffe : Jern-Kontorets Annaler, XXI., pp. I I-64, I866. Abstract in IDing. ler's Polytechnisches Journal, CLXXXV., pp. 205 and 282, 1867. Also in Kent's Mechanical Engineer's Pocket-Book.

'Pogg. Ann., Ergänzungsband II., pp. I-II 5.

${ }^{3}$ Effect of Drawing on the Elasticity of Copper Wire. J. R. Benton, Phys. REv., XIII., 4, pp. 234-245, I 901 . See also the researches of Wertheim and Pisati.

- Prof. Thos. Gray : Effect of Hardening on the Rigidity of Steel. Science, New Series, Vol. XVI., p. 337, 1902.
} 
$A$ was a strong bracket fastened firmly to the wall; the wire $B, B$ passed through a hole in $A$ and was held there by a set-screw. $C$, $C$ was an iron pipe, through which the wire hung; it was one half inch in external diameter, and $90 \mathrm{~cm}$. in length. On its lower end was soldered a chuck $D$, which was screwed up so as to grip the

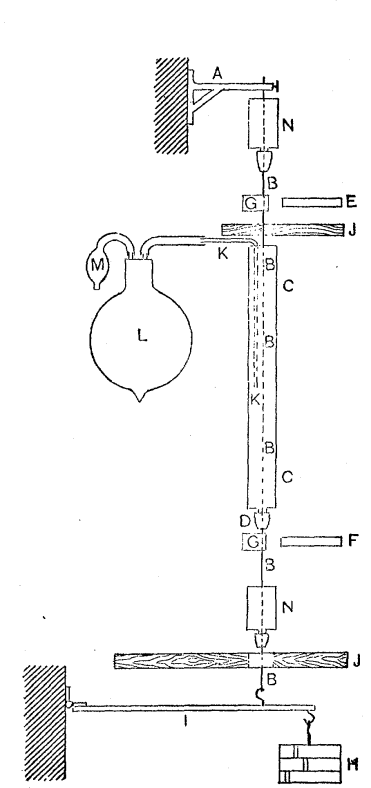

Fig. 5. wire, and at the same time close the end of $C$. The extension of the wires under varying stress was measured with micrometer microscopes, placed at $E$ and $F$; the distance between the marks on which they were sighted was about $98 \mathrm{~cm}$. When the wire was cooled with liquid air, thick frost deposited rapidly, and obscured the marks; this difficulty was overcome by fastening on the wire little air-tight vessels $G, G$, containing calcium chloride, and closed by plane glass on the side towards the micrometers. The marks were illuminated by light from a distant gas flame, focused upon them by lenses. The stretching forces were applied by the weights $H$, acting through the lever $I$, of mechanical advantage five fourths. $J, J$ were rigid shelves for supporting the micrometers. Liquid air was introduced into the pipe $C$ from the Dewar bulb $L$ by means of a force-pump arrangement $M$, devised by Dr. J. S. Shearer. $K, K$ was a glass tube, reaching to about 50 $\mathrm{cm}$. from the bottom of $C$.

In order that the points on the wire at $E$ and $F$ should have the same temperature as those within $C$, the short tubes $N, N$ were fastened on the wire. The intention was to keep them full of liquid air ; I did not succeed, however, in securing a suitable arrangement for keeping them filled; they were empty during my observations, which are accordingly subject to error to the extent that part of the wire between $E$ and $F$ was not as cold as that within $C$. Of the total length between $E$ and $F$, about eleven twelfths was within $C$. 
When the pipe $C$ was filled with liquid air, the tension of the wire being constant, it was found that the marks at $E$ and $F$ slowly moved up and down in an irregular manner, owing presumably to irregular thermal expansion and contraction in the wire above $E$, according as it was warmed by air currents from the room or cooled by the air boiling off at the top of $C$. To measure the extension under these conditions, the following procedure was adopted.

With a given load on $H$, a reading was taken at $F$; the instant it was obtained an assistant changed the load on $H$, and a reading at $F$ with the new load was taken. The time required for such a pair of readings was less than ten seconds. The difference between the two readings gives the depression of the point $F$; by taking the two readings of a pair rapidly, concordant results for the depression were obtained, although the reading for the same load fluctuated continually. Ten such pairs of readings were taken for the lower micrometer; then ten were taken similarly for the upper one; the difference of depression at $E$ and at $F$ gives the elongation of the wire between $E$ and $F$.

Apparatus for the Mudulus of Rigidity.

In researches on the modulus of rigidity the method of torsional oscillations has been employed almost exclusively, on account of its great convenience and accuracy, as compared with other methods. In my experiments, where the wire hung through a tube filled with rapidly evaporating liquid, the method was clearly impracticable; I therefore used a statical method.

The apparatus is shown in Fig. 6, which, like Fig. 5, is merely diagrammatical. The method of suspension from $A$, and the tubes $C$ and $N, N$, are the same as used for Young's modulus. To the top of $C$ was rigidly fastened

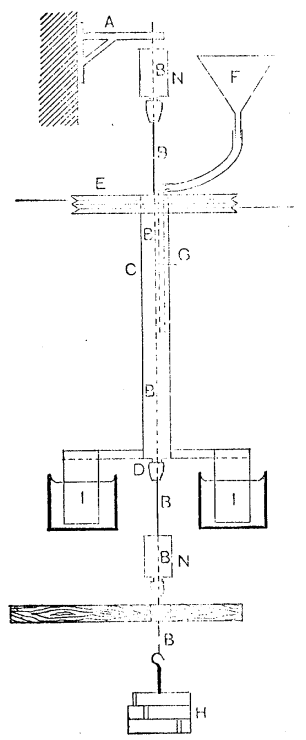

Fig. 6 . a horizontal wooden pulley $E, 24 \mathrm{~cm}$. in diameter. Liquid air was introduced into the pipe $C$ by means of a glass tube $G$, which was 
connected to a funnel $F$, supported on the pulley $E$, and free to turn with it. In order to damp torsional oscillations, the two vanes $I, I$, dipping in vessels of glycerine, were clamped to the lower end of $C$. Besides the apparatus shown in Fig. 6, there was at the top of the wire a torsion-head arrangement; and at the bottom of $C$ a system of counterpoise weights for bringing the center of gravity of $C$ below its point of attachment to the wire.

The arrangement for applying a constant twisting moment to the wire is shown on a smaller scale in Figs. 7 and 8 . Light flexible threads, $a, a$, were fastened at one end to opposite sides

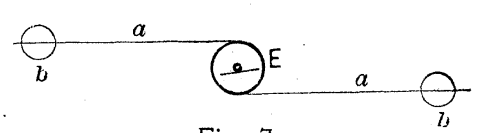
of the rim of the pulley $E$, at the other end to the walls of the room about $70 \mathrm{~cm}$. above

Fig. 7. $E$. The distance from $C$ to each wall was about 2 meters. About I meter from $C$, on each side, very light scale-pans $b, b$ were fastened on the thread. If weights were placed on these scale-pans a couple acted on the pulley $E$, by reason of the increased horizontal pull in $a, a ; E$ accordingly turned until the couple was balanced by torsion of the wire. To determine the absolute value of this couple, accurate knowledge of the various distances and of the weight of the thread would have been necessary. Since, however, the purpose of the experiments was simply to compare the torsional elasticity at $-\mathrm{I} 86^{\circ} \mathrm{C}$. with that at ordinary temperature, it was not necessary to know the absolute value of the couple, but only to be able to apply the same couple at different times.

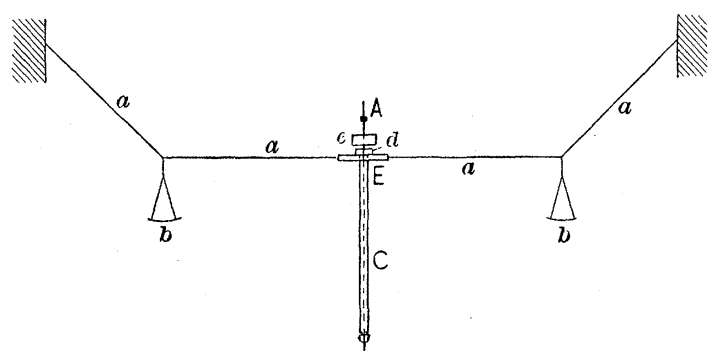

Fig. 8.

To measure the angle through which the wire was twisted a vertical plane mirror $d$ (Fig. 8) was fastened to the pulley $E$. 
Since $E$ was rigidly fastened to the pipe $C$, which in turn was fastened to the lower end of the wire, $d$ turned through the same angle as the lower end of the wire. A second vertical plane mirror $e$ (Fig. 7) was clamped to the wire itself just above the top of $C$. By means of lenses the light of a gas burner passing through a narrow slit was brought to a focus after reflection from the mirrors $d$ and $e$, so that two images of the slit were cast upon a scale $359 \mathrm{~cm}$. distant from the wire and 4 meters in length. By reading the distances through which these images were displaced by the addition of weights to the scale-pans $b, b$, the angles through which the mirrors $d$ and $e$ turned could be computed; the difference between them gives the angle of twist of the wire.

The apparatus was used in the following manner: Weights were chosen, which when placed in the scale-pans $b, b$, twisted the wire about $\mathrm{I}^{\circ}$. A set of twenty readings was made at room temperature, alternately with and without these weights in the scalepans; the position of the wire was slightly varied two or three times during the set by means of the torsion head at its upper end. Then the pipe $C$ was filled with liquid air, and the same process was repeated, using the same weights. If $R_{T}$ is the modulus of rigidity and $\alpha_{T}$ the angle of twist at temperature $T^{\circ} \mathrm{C}$., then we have

$$
R_{-186}=R_{20} \times \frac{\alpha_{20}}{\alpha_{-186}},
$$

making no allowance for thermal contraction. After the wire had returned to room temperature, another set of readings was taken.

My thanks are due to Mr. K. P. Beardslee, of Cornell University, for assistance in taking the readings for Young's modulus.

Cornell University, June, $x 902$. 